Service social

\title{
L'intervention en contexte de crise économique : le travail social avec les mineurs non accompagnés en Espagne
}

\section{Katherine Mc Crae et Marie Lacroix}

Volume 60, numéro 1, 2014

Des enfants, des couples et des familles.

URI : https://id.erudit.org/iderudit/1025132ar

DOI : https://doi.org/10.7202/1025132ar

Aller au sommaire du numéro

Éditeur(s)

École de service social de l’Université Laval

ISSN

1708-1734 (numérique)

Découvrir la revue

Citer cet article

Mc Crae, K. \& Lacroix, M. (2014). L'intervention en contexte de crise économique : le travail social avec les mineurs non accompagnés en Espagne. Service social, 60(1), 34-48. https://doi.org/10.7202/1025132ar
Résumé de l'article

Dans le cadre d'un stage international de maîtrise réalisé à Grenade, Espagne, nous avons eu l'occasion de nous pencher sur la problématique des jeunes mineurs non accompagnés et de repenser le rôle du travailleur social dans un contexte de crise économique avec une population vulnérable. La question des mineurs se pose avec beaucoup d'acuité pour les travailleurs sociaux de divers pays d'Europe à l'heure actuelle, surtout si l'on considère l'impact des mesures d'austérité dans tous les domaines des politiques sociales du social. Le présent article documente cette expérience de stage et soulève des questions pour la pratique du service social qui sont valables tant pour le Québec que pour d'autres sociétés où de plus en plus les populations immigrantes se voient reléguées à une position marginale dans les sociétés d’accueil. 


\title{
L'intervention en contexte de crise économique : le travail social avec les mineurs non accompagnés en Espagne
}

\author{
MC CRAE, Katherine \\ Titulaire de la maitrise en service social \\ Baccalauréat en travail social \\ Université de Montréal \\ LACROIX, Marie \\ Professeure agrégée \\ École de service social \\ Université de Montréal
}

\section{RESUME}

Dans le cadre d'un stage international de maîtrise réalisé à Grenade, Espagne, nous avons eu l'occasion de nous pencher sur la problématique des jeunes mineurs non accompagnés et de repenser le rôle du travailleur social dans un contexte de crise économique avec une population vulnérable. La question des mineurs se pose avec beaucoup d'acuité pour les travailleurs sociaux de divers pays d'Europe à l'heure actuelle, surtout si l'on considère l'impact des mesures d'austérité dans tous les domaines des politiques sociales du social. Le présent article documente cette expérience de stage et soulève des questions pour la pratique du service social qui sont valables tant pour le Québec que pour d'autres sociétés où de plus en plus les populations immigrantes se voient reléguées à une position marginale dans les sociétés d'accueil.

Mots clés : Mineurs non accompagnés, immigration en Espagne, travail social international, travail social et mineurs non accompagnés.

\begin{abstract}
This paper discusses an international field placement at the Master's level that occurred with unaccompanied minors in Granada, Spain. The experience allowed us to rethink the role of the social worker in a context of economic crisis with a vulnerable population. The issue of unaccompanied minors is an important one for social workers at the present time in different countries of Europe, especially considering the impact of austerity measures in all domains of social life. This article documents this field experience and raises questions for social work practice that are valuable as much for Québec as for other societies where increasingly migrant populations are being relegated to the margins of the host society.
\end{abstract}

Keywords: Non accompanied minors, immigration in Spain, international social work, social work. 


\section{INTRODUCTION}

L'immigration a connu une croissance significative depuis les dernières années en Espagne et le pays est maintenant reconnu comme l'une des portes d'entrée principales en Europe pour les immigrants provenant du continent africain. Parmi ces immigrants, il y a un nombre important de mineurs non accompagnés, phénomène ayant fait son apparition au début des années 1990. Les mineurs non accompagnés constituent un groupe extrêmement vulnérable et, depuis 2002, leur nombre n'a cessé de croître (Observatorio de la infancia en Andalucia [OIA], 2012) sauf, exceptionnellement en 2008, lorsqu'il y a eu une légère diminution dans la région de l'Andalousie (OIA, 2012). Inévitablement, leur situation préoccupe les services sociaux et constitue un défi de taille pour les intervenants sociaux surtout dans le contexte de crise économique qui sévit présentement en Espagne.

En janvier 2013, il y avait plus de 300 enfants qui vivaient sous tutelle de la communauté autonome province d'Andalousie dans des centres de protection. Dans ces centres, ils reçoivent des services qui répondent, à différents degrés, à leurs besoins économiques, physiques, sociaux, émotionnels et d'estime (OIA, 2012; Cruz Roja Española [CRE], 2011). À leur arrivée, la plupart sont reconnus comme des mineurs en situation de détresse ou d'abandon et ils sont placés sous la tutelle du système de protection tel que prévu par la loi.

Dans le cadre d'un stage de maîtrise en service social à l'Université de Montréal et en partenariat avec l'Université de Grenade en Espagne, nous avons eu l'occasion de nous pencher sur la problématique des jeunes mineurs non accompagnés et de repenser le rôle du travailleur social dans un contexte de crise économique avec une population vulnérable. Le stage s'est déroulé à la Croix-Rouge de Grenade. En Espagne, la Croix-Rouge est l'un des principaux acteurs de l'intervention auprès des mineurs non accompagnés, mis à part les centres de protection dans lesquels vivent les jeunes. D'une durée de quatre mois, le stage a débuté en octobre 2012 pour prendre fin en janvier 2013. La question des mineurs se pose avec beaucoup d'acuité pour les travailleurs sociaux de divers pays d'Europe à l'heure actuelle, surtout si l'on considère l'impact des mesures d'austérité dans tous les des politiques sociales. Le présent article documente cette expérience de stage et soulève des questions pour la pratique du service social qui sont valables tant pour le Québec que pour d'autres sociétés où de plus en plus les populations immigrantes se voient reléguées à une position marginale dans les sociétés d'accueil.

\section{Projet de stage initial}

La Croix-Rouge espagnole travaille au développement de ressources de transition ou de ressources alternatives au système de protection de l'enfance pour les mineurs non accompagnés. Les interventions sont différentes d'une ville à l'autre, mais l'objectif demeure le même : protéger les enfants vulnérables ou à risque en comblant leurs besoins et en assurant la protection de leurs droits. À Grenade, la Croix-Rouge dispose d'un appartement d'émancipation pour les mineurs non accompagnés qui viennent d'atteindre leur majorité puisque, à dix-huit ans, les jeunes doivent quitter les centres de protection. 
Le projet de stage avait été planifié avant notre arrivée dans le milieu de stage, la CroixRouge de Grenade, suite à une recension des écrits qui sera brièvement présenté dans les paragraphes suivants. Le projet de stage prévoyait une intervention de groupe jumelé à une approche en réseau, qui permettrait le développement d'un réseau social entre les mineurs non accompagnés. Plus précisément, le but du projet était de développer un réseau social de soutien entre les mineurs non accompagnés. L'intervention ciblait les objectifs suivants : briser l'isolement, favoriser l'entraide et le soutien ainsi que l'échange d'informations et d'expériences. De ces objectifs généraux découlaient de nombreux objectifs spécifiques tels que la création du lien de confiance, la prise de conscience du réseau social, l'identification des émotions ressenties, etc.

À première vue, le projet prévu correspondait bien aux objectifs du programme de la CroixRouge. Dans un rapport, l'organisme précise qu'il est nécessaire de développer des centres d'hébergement ou de transition qui répondent aux besoins spécifiques de cette population bien différente de celle des centres de protection ou d'aide immédiate du gouvernement espagnol (CRE, 2006). L'organisme mentionne qu'il faut travailler les besoins à long terme. Toutefois, une mise en contexte de la situation en Espagne et des mineurs non accompagnés s'impose avant de développer davantage sur le stage.

\section{L'immigration en Espagne}

L'Espagne a longtemps été un pays d'émigration vers le nord de l'Europe pour les travailleurs ou vers les pays de l'Amérique latine lors de la période coloniale et de la dictature du général Franco. Cependant, l'Espagne s'est transformée en pays d'accueil pour les immigrants de façon significative vers les années 1980 (Agrela, 2002 ; Alscher, 2005) et, depuis quelques années, est devenue une porte d'accès à l'Europe. Du fait de sa situation géographique, l'Espagne est un lieu de destination ou de transit pour les migrants qui proviennent du continent africain (Alscher, 2005). Cela justifie les politiques restrictives qui sont mises en place par l'Espagne qui prône une politique de fermeture et axée sur le contrôle des flux migratoires plutôt que sur l'intégration de ses immigrants. Comme il s'agit d'un pays où la question de l'immigration est récente, les services aux immigrants demeurent encore précaires. L'Espagne est à l'opposé des pays comme l'Allemagne et le Royaume-Uni où le processus est centré sur les demandes d'asile. En effet, l'Espagne conserve une approche classique où les enfants, tout comme les adultes, sont considérés comme des immigrants avec des motifs économiques. Par exemple, la majorité des immigrants qui veulent obtenir la résidence doivent avoir un contrat de travail (Senovilla, 2007).

Dans le cadre de son adhésion à l'Union Européenne en 1980, l'Espagne développe sa première politique d'immigration en 1985 (Moffette, 2009). Dès le début cette dernière vise plutôt des éléments administratifs, soit le contrôle du marché et la sécurité publique (Agrela, 2002). À partir de 1993, la question de l'intégration sociale des immigrants commence à se poser dans les milieux politiques. Les services sont toutefois orientés vers les immigrants qui résident légalement en Espagne et excluent toute personne ayant un statut irrégulier (Agrela, 2001). En 1996, le travail social se fraye un chemin afin de participer au développement des services spécifiques aux immigrants. Ces services, quoique très limités, sont axés sur 
l'orientation et l'accès aux ressources : enseignement, santé et sécurité sociale. Agrela (2001) conclut qu'en 2001, malgré les progrès dans la politique d'immigration, les lois et les règlements sont encore largement orientés par des questions administratives.

\section{Facteurs socio-démographiques de la migration des mineurs}

Les mineurs non accompagnés ne constituent pas un bloc homogène; ils représentent une diversité d'âges, de langues et d'origines. Néanmoins, pour le cas de l'Espagne, la majorité d'entre eux est âgée de 15 à 17 ans et, vu la proximité géographique, provient du Maroc (OIA, 2009). En 2008, les centres de protection de l'Andalousie ont reçu plus de 900 mineurs non accompagnés dont $93 \%$ étaient des garçons et 84,6 \% des jeunes en provenance du Maroc (OIA, 2009), représentant 18,3\% des arrivées au pays (OIA, 2012). Alors que la majorité des mineurs non accompagnés en Espagne sont des garçons, il se trouve que les jeunes filles sont plus vulnérables que les garçons. En effet, dès leur arrivée, elles sont souvent impliquées dans des réseaux d'exploitation sexuelle de mineurs ou de travailleuses domestiques (HCNUR, 2009). Comme elles se retrouvent au sein de réseaux clandestins, elles ne bénéficient d'aucune protection prévue par la loi.

Les motifs qui incitent un jeune à quitter son pays sont nombreux : situations de conflit dans le pays, exploitation, situation économique précaire, exclusion, violence physique ou sexuelle et traite (Ayotte, 2000 ; Senovilla, 2007). L'absence de formation professionnelle, l'accès limité aux ressources de base telles que la santé et l'éducation, la résistance aux changements sociaux et l'incapacité de l'État à protéger les personnes les plus vulnérables (Jimenez, 2007) sont d'autres facteurs importants. La précarité des familles au Maroc, au cœur de cette migration, est liée à de nombreux problèmes structurels, notamment à l'exode massif des campagnes vers les villes dans les années 1970, qui a mené à une urbanisation catastrophique et au développement de nombreux quartiers défavorisés ; c'est le cas, par exemple, de Tanger et de Tetuan. Ces villes ne disposent toujours pas des ressources ou des infrastructures nécessaires pour répondre aux besoins de tous leurs habitants. L'impact de cette migration interne s'est fait sentir sur les rôles sociaux qui ont été bouleversés, alors que de nombreuses femmes doivent maintenant travailler dans des industries et des entreprises étrangères agroalimentaires ou de textile. Même si certains hommes restent à la maison parce que sans emploi, ils n'assument pas pour autant les tâches domestiques (Jimenez, 2007). Ainsi, de nombreux enfants sont laissés à eux-mêmes ou à leurs frères et sœurs aînés. Peu importe les motifs ou le profil, la plupart des enfants qui immigrent en Espagne cherchent une vie meilleure (Suárez Navaz, 2004).

\section{Les besoins, les services et les obstacles}

Selon Wouk et al. (2006) les jeunes arrivent dans des conditions variées et leurs besoins, liés à ceux de l'enfance, englobent tous les aspects de la vie : I'hébergement, la nourriture, les soins de santé, l'éducation et la présence d'un adulte pour les guider dans leur vie quotidienne. Une fois sous le système de protection de l'enfance, les jeunes sont orientés vers des centres spécifiques aux mineurs non accompagnés ou vers des centres communs au service de protection de l'enfance, selon la région dans laquelle ils se trouvent et l'espace disponible dans les différents centres (Senovilla, 2007). Ils auront éventuellement accès au système d'éducation 
pour les niveaux de scolarisation obligatoires de l'Espagne. Néanmoins, ils sont confrontés à de nombreux obstacles en ce qui concerne la formation professionnelle, les études postsecondaires ou les soins de santé. Selon Senovilla (2007), la plupart des problèmes de santé mentale sont traités dans les centres d'accueil ou des ressources spécialisées pour les mineurs non accompagnés puisque le réseau public n'offre pas de tels services.

Les objectifs économiques que se sont donnés les mineurs et qui caractérisent leur migration ont pour conséquence qu'ils vivent une grande pression financière. En effet, leur projet migratoire ne consiste pas à fréquenter l'école, mais à gagner rapidement de l'argent pour rembourser la dette du parcours migratoire et envoyer de l'argent à leur famille restée au pays (Senovilla, 2007). Cependant, plusieurs seront déçus, car le système de protection de l'enfance ne leur permet pas de travailler avant l'âge de 16 ans (HCNUR, 2009). Cette contrainte aura un impact sur l'intervention alors que d'autres besoins devront être mis de côté; ce qui est central dans leur vie est une aide dans la gestion des documents qui leur permettront de travailler et d'acquérir des compétences pratiques. (Setien et Barcelo, 2007). Ainsi, pour les mineurs non accompagnés qui ne sont pas protégés par le système ou qui décident d'eux-mêmes de fuir la tutelle pour travailler, leur situation financière est extrêmement précaire. Selon Setien et Barcelo (2007), si les services ne répondent pas au besoin de recherche d'emploi, les jeunes se découragent et sombrent dans le désespoir. C'est à ce moment que les risques de s'adonner à des activités délinquantes ou illégales, ou de se retrouver dans des réseaux de traite ou d'exploitation, augmentent (Senovilla, 2007).

Ils sont confrontés à d'autres obstacles face à l'intégration sociale, dont la discrimination figure parmi les plus importants (Kwok et Tam, 2009). Que ce soit à l'école ou dans le milieu de l'emploi, Kowk et Tam (2009) affirment que les jeunes ont le sentiment d'être traités de façon différente des autres enfants de la société d'accueil. Selon Agrela (2002), cette discrimination découle des préjugés qui sont véhiculés dans les médias et l'opinion publique, qui exagèrent les cas d'illégalité et qui mettent en lien l'immigration, l'illégalité et le crime, créant un sentiment d'insécurité au sein de la population espagnole. Mais pour le jeune, l'insécurité, la solitude et l'isolement caractérisent son arrivée dans un pays inconnu; cela aura un impact majeur sur son sentiment d'intégration à la société d'accueil (Kohli, 2006; 2007). Les mineurs se retrouvent sans repères sociaux ou familiaux et, pour ceux qui ont grandi dans un milieu communautaire où la communauté est une famille, la perte de ce grand réseau peut être encore plus difficile à supporter (Montgomery, 2002). D'ailleurs, plusieurs recherches ont démontré à quel point la famille proche joue un rôle primordial dans le processus d'immigration (Vatz Laaroussi, 2009; Bergeron et Potter, 2006).

Dans la plupart des situations, il est important de prendre en considération qu'il ne s'agit pas d'un départ volontaire. Tel que mentionné précédemment, les motifs sont nombreux et liés à l'insécurité et à des conditions de vie difficiles, alors que, pour certains, il s'agit du projet de leurs parents ou de leur communauté. En conséquence, le contexte d'intégration ne sera pas le même selon les conditions de départ. Le statut irrégulier se pose donc comme obstacle majeur auquel est confronté le jeune (Ayotte, 2000). Outre les besoins primaires, il est primordial pour le jeune de régulariser son statut sans quoi il sera privé de nombreux services. Ces jeunes vivent dans des centres de protection jusqu'à 18 ans où leurs besoins primaires sont comblés; 
toutefois, dès qu'ils atteignent 18 ans, la société attend d'eux qu'ils vivent de façon autonome, ce qui signifie apprendre à faire un budget, régulariser son statut, apprendre à cuisiner, trouver un travail, trouver un logement, etc. (Jimenez, 2007; Montgomery, 2002).

\section{Intervention en fonction des besoins}

Le programme «Separated Children in Europe »(UNHCR, 2009), dans sa déclaration de bonnes pratiques, énonce une réglementation au niveau international afin de protéger les droits de ces enfants. Parmi les principes qui y sont soulignés, se retrouvent: l'intérêt supérieur de l'enfant, la non-discrimination, le droit de participer, le respect de l'identité culturelle, la confidentialité, l'information, la coopération entre les organismes qui travaillent avec eux, la formation de professionnels dans le milieu, la durabilité et le temps. Enfin, des principes semblables à ceux qui sont utilisés dans le système de protection de la jeunesse et devraient être pris en compte dans l'intervention auprès de cette population. Ces principes directeurs se conjuguent très bien avec une approche structurelle et de défense des droits.

Pendant le processus d'intervention, plusieurs chercheurs (Kohli, 2007; Hopkins et Hill, 2010; Wade, 2011) soulignent l'importance de considérer la spécificité de la situation de chaque enfant et surtout, étant donné l'hétérogénéité du groupe, de tenir compte de l'expérience prémigratoire qui aura un impact sur l'identité, les besoins, les comportements et la perspective qu'ont les jeunes de leur situation, tributaire des difficultés d'intégration vécues dans le pays d'accueil (Wade, 2011). Dans cette perspective Wade (2011) suggère une approche davantage centrée sur le jeune où l'intervenant est à l'écoute des besoins spécifiques de chacun.

À l'arrivée du jeune, les intervenants se concentrent sur l'information de base par exemple : l'âge, l'année de scolarité, la santé, le nombre de frères et sœurs, etc., et ont tendance à attendre que le jeune soit installé et que sa situation soit plus stable avant de poser des questions plus complexes sur le passé et les raisons de l'exil (Wade, 2011). Dans ce sens, Hopkins et Hill (2010) expliquent qu'il faut comprendre les priorités des besoins du jeune dans un continuum temporel : répondre aux besoins immédiats ; aux besoins associés au futur, ce qui inclut les besoins socioculturels tels que le sport, les loisirs, les groupes d'entraide, les groupes religieux, etc., avec comme objectif de créer un réseau social et de lier de nouvelles amitiés ; en dernier lieu, aborder le passé. C'est lors de cette dernière étape qu'il y a centration sur l'état psychologique du jeune incluant les besoins émotionnels, le retour sur le passé et le lien avec sa culture d'origine (Hopkins et Hill, 2010).

L'isolement, la peur et le silence, souvent présents chez les mineurs non accompagnés, sont des éléments qui requièrent une intervention appropriée et équilibrée. Le silence peut procurer à la fois un sentiment de sécurité pour le jeune, mais peut aussi devenir un fardeau (Kohli, 2007). Alors que, pour certains, le silence permet de gérer les atrocités vécues et, avec le temps, il peut permettre de guérir certaines blessures psychologiques ou même d'oublier certains éléments de leur histoire. Pour d'autres, le silence devient trop lourd et ils ne peuvent gérer seuls ce qu'ils ont vécu, sans pour autant vouloir partager leur expérience. Cela s'explique par plusieurs motifs: soit la peur de parler parce que les personnes qui ont planifié leur parcours migratoires leur ont dit de garder le secret afin de ne pas contredire leur histoire ; soit parce 
qu'ils ont peur d'être jugés d'avoir voyagé avec de faux papiers ou parce qu'ils ne connaissaient pas leur âge exact, etc. (Kholi, 2007). Il est donc primordial pour l'intervenant de faire preuve d'ouverture d'esprit, de ne pas faire sentir aux jeunes qu'ils sont jugés ou questionnés quant à la véracité de l'histoire et, surtout, il est important de respecter le rythme de chaque jeune quant à la divulgation de son présent ou même de son passé.

\section{L'approche interculturelle auprès des immigrants}

Afin de mettre en place une intervention appropriée auprès des mineurs non accompagnés, il faut tenir compte de tous les éléments ayant un impact de près ou de loin sur leur situation. À cet effet, Verbunt affirme que « l'action la plus efficace est donc celle qui s'attaque à la fois aux causes structurelles et culturelles, tout en prenant en compte la singularité des comportements individuels » (Verbunt, 2009, p. 39). L'approche interculturelle permet des pratiques plus adaptées à un contexte multiculturel, favorisant une meilleure communication, une compréhension et une tolérance de la diversité (Cohen-Émerique, 1993; Legault, 2000). Elle permet à l'intervenant de se distancier en réfléchissant sur soi-même pour prendre conscience de sa propre culture et s'interroger sur son système de valeurs, ses préjugés et ses motivations (Cohen-Émerique, 2000) ; elle permet de pénétrer le système de l'autre et, dans un contexte de médiation et de négociation, cherche à mettre fin aux conflits à l'aide du dialogue et de l'échange (Cohen-Émerique, 1993). Pour Fong (2004) et Potocky-Tripodi (2002), la compétence culturelle est basée sur la compréhension de la diversité des cultures et l'adaptation de l'intervenant au contexte. Selon une étude réalisée auprès de différents organismes dans la région d'Andalousie, les activités qui favorisent les échanges culturels, autant dans les centres de protection que dans les écoles, permettent de prévenir les visions racistes et xénophobes (OIA, 2006). Selon Fong (2004), l'intersectionnalité prend tout son sens dans l'intervention auprès des immigrants car, au-delà de la culture, il faut tenir compte de multiples variables: race, genre, classe, religion, statut légal, environnement social, etc. Dans un contexte de pluralité aussi significatif que celui de l'Espagne et des centres de protection, ces différentes formes de pratiques interculturelles peuvent être pertinentes pour guider et orienter l'intervention auprès des mineurs non accompagnés.

\section{L'approche structurelle}

Lors du stage, l'analyse et l'intervention de façon globale avec les jeunes ont été guidées par l'approche structurelle. L'approche structurelle s'insère dans les perspectives et les pratiques radicales du service social (Moreau, 1987). De façon plus précise, cette approche permet de relier à l'action les idéologies socialistes et certains principes marxistes. Cela signifie que cette approche tient compte dans son analyse de la réalité sociale et de l'impact des déterminants socio-économiques (Lévesque et Panet-Raymond, 1994). Cette approche prend tout son sens dans le contexte actuel de l'Espagne qui, dans les dernières années, a connu de nombreux bouleversements dus à la crise économique, entraînant des impacts significatifs et visibles sur toutes les sphères sociales. Les mesures d'austérité, soit la réduction des dépenses publiques imposée par le gouvernement espagnol actuel, entraînent des coupures dans les services sociaux, l'éducation et la santé, touchant la majorité des citoyens de l'Espagne, mais davantage ceux déjà victimes d'exclusion sociale. Ainsi, une approche critique nous paraît primordiale dans 
l'intervention qui permettra aux jeunes d'être reconnus et les encouragera à se mobiliser. De plus, l'approche structurelle propose des techniques d'intervention qui se lient très bien au cadre d'analyse et aux objectifs visés par l'intervention qui sont l'augmentation du pouvoir de la personne (empowerment), la défense des droits des individus et des collectivités, le questionnement de l'idéologie dominante, la matérialisation et la collectivisation des problèmes (Lévesque et Panet-Raymond, 1994; Moreau, 1987).

\section{Les impacts de la crise économique sur la situation des mineurs non accompagnés}

Dans les dernières années, de nombreux centres de protection des mineurs non accompagnés, qu'ils soient privés ou publics, ont dû fermer leurs portes et les centres qui demeurent ouverts sont sous-financés. II en résulte que les ressources d'hébergement sont surchargées et les services offerts limités. Ainsi, même si de nombreux intervenants connaissent les besoins spécifiques des mineurs non accompagnés, ils ont peu de ressources financières ou humaines pour répondre aux besoins. À l'instar des centres de protection, c'est aussi le cas des organismes qui offrent des services complémentaires comme la Croix-Rouge. II résulte de cette situation de pénurie l'adaptation à une réalité changeante tel qu'elle est valorisée par l'approche structurelle. L'intervention cible les besoins urgents des mineurs non accompagnés et l'intervention à long terme devient secondaire comme la décrit la théorie temporelle de Hopkins et Hill (2010). Il est devenu évident que lorsque les jeunes vivent en centre de protection, les besoins présents sont comblés (se loger, se nourrir, se vêtir, etc.) et le travail au niveau des besoins futurs (émancipation, régularisation du statut, préparation au milieu du travail) n'est que très peu abordé. Ainsi, lorsque les jeunes sont contraints de quitter le centre de protection, à l'âge de 18 ans, ils se retrouvent une fois de plus à la case départ, à la première phase du besoin présent. Étant donné le manque de ressources humaines, le travail au niveau du passé est très peu abordé.

L'impact majeur de la crise, il faut le rappeler, est sur l'emploi. Les économistes affirment que l'économie espagnole doit croître d'environ $2 \%$ avant de pouvoir créer des emplois soutenus, ce qui ne se produira pas avant la fin de 2013, voire de 2014 (Castello, 2013). Même s'il n'existe aucune statistique sur le taux de chômage des mineurs non accompagnés, le simple fait qu'ils soient des jeunes et des travailleurs peu qualifiés permet d'émettre l'hypothèse, soutenue par les statistiques sur les jeunes en général au pays, que ce taux dépasse les $40 \%$. Tout cela génère beaucoup de frustration. Lors du stage, la difficulté d'obtenir un emploi a été un problème récurrent pour tous les jeunes non accompagnés qui atteignent l'âge de 18 ans et qui se retrouvent à la rue sans aucune ressource financière.

Étant donné les besoins urgents auxquels font face ces jeunes et qui diffèrent de ceux identifiés dans le projet de stage, et compte tenu de la conjoncture économique en Espagne et du manque d'intervenants impliqués dans le projet, des restrictions dans le temps, soit quatre mois de stage dont le premier fut consacré à la connaissance du milieu, le projet a dû être adapté à la situation du pays et a donc pris une tournure complètement différente de celle prévue dans le projet de stage initial. Ces changements dans le projet de stage sont tout à fait cohérents avec l'approche structurelle et Lévesque et Panet-Raymond (1994) précisent d'ailleurs l'importance de s'adapter à un contexte qui est en mouvement constant autant dans 
l'analyse que dans l'intervention. Ainsi, le projet de stage a plutôt dévié vers un projet individualisé d'aide à la recherche d'emploi.

\section{Discussion stage et analyse}

Une fois sur place la réalité du terrain a fait que des réajustements ont été nécessaires dans le projet de stage, ce qui constitue en soi un apprentissage important pour la pratique. Tel que mentionné précédemment, les impacts de la crise économique ont eu une influence sur les besoins des mineurs non accompagnés et, par le fait même, sur les services offerts. Ce n'est que sur place que ces changements ont été constatés puisque ces événements récents étaient et sont encore très peu documentés. Mon projet est donc devenu un appui individualisé, pour la rédaction de curriculum vitae, le développement de stratégies de recherche d'emploi, les pratiques d'entrevue, et le soutien dans les inscriptions à des cours ou à des formations pratiques. Le projet s'est adressé à huit jeunes de l'appartement d'émancipation de la CroixRouge. Tous des garçons, ils avaient 18 ans; sept étaient d'origine marocaine et un originaire du Ghana et ils provenaient de différents centres de protection d'Andalousie. La plupart avaient un niveau d'espagnol assez élevé, étant arrivés vers l'âge de 16 ans et ayant été scolarisés en espagnol. Les deux jeunes qui avaient le plus de difficulté ont été orientés vers des cours d'espagnol aux adultes. Ils avaient des parcours scolaires et des formations variés, allant de la mécanique à la coiffure. Les jeunes étaient suivis chaque semaine, avec au moins deux rencontres individuelles axées sur la recherche d'emploi et les besoins de base, ainsi qu'une rencontre de groupe pour le suivi de la vie de groupe en appartement. Certains étaient en formation, d'autres en stage d'essai, mais pour ceux qui étaient en recherche d'emploi ou de stage, ils devaient se présenter tous les jours pour faire le suivi des entreprises visitées. Les intervenants de la Croix-Rouge avaient souvent à prendre contact avec les différents employeurs afin de déconstruire certains mythes et préjugés face à ces jeunes immigrants. Dans ces cas, l'approche interculturelle prenait tout son sens. Basée en effet sur l'ouverture, cette approche favorisait une meilleure communication et compréhension autant auprès des employeurs que des jeunes. Les intervenants de la Croix-Rouge deviennent le point d'ancrage pour ces jeunes qui essaient de s'intégrer dans leur nouvelle société d'accueil. En ce sens, comment parler d'intégration auprès d'une population dont on ne reconnaît pas l'existence sociale et dont les lois poussent plutôt à la restriction des droits qu'à l'intégration. Malgré les efforts des intervenants et des jeunes, le problème d'emploi persiste, tant pour les Espagnols que pour les immigrants. Pendant le stage de quatre mois, des huit jeunes, seulement deux ont réussi à obtenir leur permis de travail et un autre commençait un stage en attente d'un contrat de travail qui permettrait de faire la demande du permis de résidence et de travail. Les permis sont régis selon la loi organique 4/2000 du 11 janvier pour les droits et libertés des étrangers en Espagne et l'intégration sociale. Lorsqu'ils sont mineurs, les jeunes obtiennent un permis de séjour, permis qui se renouvelle chaque année et qui leur permet sans souci de résider sur le territoire espagnol jusqu'à l'âge de 18 ans. À ce moment, ils sont pris en charge par l'État qui s'occupe de répondre à leurs besoins de base par l'entremise des centres de protection dont très peu sont spécialisés dans les services aux jeunes immigrants, ils ont aussi accès à l'éducation et aux formations professionnelles. Ils peuvent pendant ce temps faire la demande de résidence permanente, un processus long qui exige souvent plus de deux ans sur le territoire espagnol (Ministerio de Empleo y Seguridad social, 2013). Ce qui signifie que très peu de 
mineurs non accompagnés parviennent à obtenir cette résidence permanente. Une fois la majorité atteinte, la situation se complique pour eux, les jeunes doivent travailler pour subvenir à leurs besoins et, pour travailler, ils doivent avoir un permis de travail et, pour avoir ce permis, ils doivent avoir un emploi ou un contrat de travail. Ces contrats sont extrêmement difficiles à obtenir pour différentes raisons: manque de qualification, xénophobie et, surtout, manque d'emplois. Cela entrave de façon significative leur intégration et, d'autant plus, le sentiment d'appartenir à la société d'accueil.

De cette problématique découle de nombreux problèmes: pas d'emploi, pas d'aide sociale, pas de résidence de séjour ou de travail, pas d'accès au système de santé, pas de logement, etc. Les actions du gouvernement sont axées sur les coupures et non sur l'aide à l'employabilité ce qui a pour effet que les intervenants se retrouvent dépourvus et les jeunes découragés. Les services auprès des mineurs non accompagnés semblent se détériorer au détriment des intervenants et des mineurs non accompagnés. L'État est obligé d'offrir des services aux mineurs non accompagnés, étant donné les différentes lois qui protègent les droits des enfants, les services offerts dans les centres de protections sont toutefois restreints aux besoins de base : se loger, se nourrir, se vêtir. Pourquoi investir dans l'avenir de ces jeunes qui ne seront plus de sa responsabilité dans un avenir rapproché ? En effet, une fois que ces jeunes atteignent la majorité, l'État n'a plus aucune responsabilité envers eux. Plutôt que d'investir dans des organismes qui aident les mineurs non accompagnés une fois la majorité atteinte, des subventions espagnoles sont octroyées à des organismes au Maroc afin de mettre en place des mesures de retour et d'intégration dans le pays d'origine. Ainsi, on peut supposer que les politiques sont orientées vers le retour des mineurs à leur pays d'origine plutôt que sur leur intégration en Espagne (Agrela, 2001).

Contrairement au Canada, où les services sociaux sont gérés par l'État ainsi que par des organismes communautaires permanents, la majorité de l'intervention sociale en Espagne est réalisée par des organismes non gouvernementaux tels que la Croix-Rouge et Caritas. Le cas de l'Espagne est particulier, puisque les organismes non gouvernementaux (ONG) sont arrivés tardivement en Espagne et ils ont pris en charge le volet de l'intervention sociale (Gomez Gil, 2005). Le mandat des ONG est d'offrir une aide immédiate et ponctuelle, et ce, avec une grande population. Sont donc privilégiés des modèles d'intervention à court terme tel que l'intervention de crise, ce qui engendre des lacunes importantes dans la prestation des services sociaux. Les approches telles que l'empowerment et le travail structurel sont donc très peu utilisées puisqu'elles valorisent le travail à long terme. Tel que mentionné précédemment avec l'exemple de l'appartement supervisé, l'objectif de la Croix-Rouge est d'aider les jeunes à se trouver un emploi afin de libérer des places le plus rapidement possible et donc, d'accueillir le plus grand nombre de jeunes. Deux raisons justifient cela, la première mentionnée précédemment étant le mandat d'assistance immédiate et la deuxième, les subventions. Les subventions que reçoit la Croix-Rouge dictent en quelque sorte ses activités c'est-à-dire que ses interventions et leurs statistiques doivent correspondre à des activités spécifiques à réaliser en début d'année, et ce, même si les besoins évoluent au courant de l'année ce qui entre en contradiction avec l'approche structurelle qui, elle, prône une adaptation aux changements. 
Sur le plan organisationnel, la Croix-Rouge est un organisme international avec des principes directeurs qui lui permettent de conserver une certaine cohérence dans tous les pays où elle intervient. II y a sept principes qui orientent les actions de la Croix-Rouge, autant en Espagne que dans les autres pays. Parmi ces principes, le principe de neutralité, défini comme suit: «Afin de garder la confiance de tous, elle [la Croix-Rouge] s'abstient de prendre part aux hostilités et, en tout temps, aux controverses d'ordre politique, racial, religieux ou philosophique »(Cruz Roja, 2012). En général, les principes peuvent apparaître tout à fait adéquats pour un organisme international présent dans la majorité des pays en tant qu'acteur neutre dans des régions de conflit ou de désastre. Toutefois, dans le contexte de l'Espagne où il s'agit d'une entité avec un rôle important dans les services offerts aux mineurs non accompagnés, ce principe devient alors en contradiction avec le rôle du travailleur social et la défense des droits. La Croix-Rouge et les intervenants qui représentent l'organisme évitent de se prononcer sur des sujets controversés tels que la question du rapatriement des mineurs non accompagnés et l'organisme n'appuie aucune revendication. Cette situation freine donc toute activité politique à l'extérieur de l'organisme.

Toujours au plan organisationnel, la bureaucratie a été un autre facteur nuisible pendant le stage. Alors que la bureaucratie est inévitable dans un système institutionnel tel que la CroixRouge, un problème surgit lorsqu'elle devient trop lourde et qu'elle nuit à l'intervention, comme c'est d'ailleurs le cas dans plusieurs milieux institutionnels du Québec. Les intervenants de la Croix-Rouge sont surchargés de tâches bureaucratiques et de gestion ce qui alourdit de façon significative leur travail en général. II y a donc peu de contact direct avec les jeunes. Par exemple, l'intervenante qui supervisait mon stage devait non seulement s'occuper des rapports, des statistiques, des notes évolutives, mais en plus de gérer les dépenses liées à l'appartement. La plupart des intervenants de la Croix-Rouge doivent préparer des ateliers, qu'ils ne réaliseront pas puisqu'ils seront animés par des bénévoles. Dans ce sens, les intervenants doivent se charger de former et de recruter les bénévoles. Ainsi, les intervenants pourraient être qualifiés comme des gestionnaires de projet et les bénévoles seraient les intervenants. Ce qui nous amène à nous questionner sur les limites de l'intervention des bénévoles. Bien que certains bénévoles soient des étudiants dans le domaine social, la majorité n'est pas nécessairement formée pour répondre aux besoins des jeunes immigrants.

\section{CONCLUSION}

Peu importe le motif ou la situation de départ, les jeunes arrivent tous en Espagne avec l'objectif d'améliorer leur vie. Ils rêvent de jouer dans une ligue professionnelle de soccer ou de travailler et de devenir riches. Plusieurs seront déçus et réaliseront rapidement que la réalité n'est pas celle qu'ils avaient projetée au début du parcours migratoire. La crise économique a engendré de nombreux défis et enjeux pour les mineurs non accompagnés et les intervenants en Espagne. Les mesures d'austérité et la pénurie d'emplois étaient et demeurent les problèmes majeurs du contexte social. L'approche structurelle permet une analyse des impacts significatifs de la crise économique sur les services sociaux et les besoins des mineurs ainsi qu'une méthodologie d'intervention tout à fait appropriée au contexte. Pourtant, confronté à la nature de l'organisme et aux ressources financières et humaines limitées, le pouvoir d'action est très limité. Cette situation s'applique aussi à de nombreux groupes vulnérables qui sont touchés par 
la crise économique. Dans ce contexte, les mouvements sociaux prennent une place grandissante dans le pays et les revendications deviennent plus insistantes. Parmi les mouvements sociaux importants en Espagne, on retrouve celui des étudiants qui revendiquent une éducation accessible à tous, celui des sans-papiers dont le slogan est «Aucun humain n'est illégal » et celui contre la privatisation des soins de santé. Cependant, en raison du contexte du stage, une méthodologie d'intervention axée sur des revendications et sur la participation aux mouvements sociaux a été impossible. Ces mouvements sont basés sur une vision structurelle et revendicatrice, ils dénoncent les mauvaises pratiques du gouvernement qui freinent, de manière importante, l'accessibilité aux études, l'intégration des immigrants et l'accès aux soins de santé. Cela étant dit, dans un contexte différent, la mobilisation et l'action collective deviennent un levier d'action pertinent qui permettrait aux différents groupes de se sentir reconnus et entendus, et ce, autant en Espagne qu'ici au Québec. Comme le soutiennent Bailly et Licata (2011), les actions collectives permettent aux personnes de retrouver une image positive et une confiance en soi, gage d'un avenir meilleur.

II serait aussi fort utile de développer un outil qui permettrait d'informer les jeunes et les employeurs et de déconstruire certains mythes qui perdurent: par exemple, plusieurs employeurs croient que le contrat de travail les engage à employer le jeune durant la durée totale du contrat alors que c'est faux. Comme tout employé, si le jeune ne répond pas aux besoins de l'employeur, il peut être congédié. Cet outil aurait été d'une grande utilité à la fois pour les intervenants, les jeunes à la recherche d'emploi et les employeurs, mais aurait nécessité un délai plus long que celui permis par le stage. En effet, pour construire un bon outil, il aurait fallu le réaliser en collaboration avec les différents acteurs impliqués: ministère du Travail, ministère de I'Immigration et région d'Andalousie. Les informations recueillies: la définition d'un contrat de travail, les législations, les informations sur le permis de travail, et les mythes demeureront tout de même dans les mains des superviseurs en espérant qu'il y aura d'autres stagiaires qui auront le désir de poursuivre la mise au point de cet outil.

Les besoins sont criants, mais l'emploi est prioritaire sans quoi les jeunes ne peuvent obtenir le renouvellement du permis de résidence. Toutefois, les deux jeunes qui ont trouvé un emploi sont une source d'espoir pour les autres jeunes et démontrent que la persévérance peut donner des résultats positifs. En effet, il ne semble pas y avoir de solution facile dans un contexte économique aussi difficile, la persévérance et la détermination sont les deux éléments qui ont mené à des résultats positifs pour les jeunes. Avec le temps, ils ont su créer des contacts auprès d'employeurs après plusieurs visites et démontrer leur potentiel grâce à des stages non rémunérés.

MC CRAE, Katherine

Titulaire de la maîtrise en service social Baccalauréat en travail social Université de Montréal

LACROIX, Marie Professeure agrégée École de service social Université de Montréal 


\section{BIBLIOGRAPHIE}

Ayotte, W. (2000). Separated children coming to Western Europe: Why they travel and how they Arrive, Londres, Save the Children.

Agrela, B. (2001). «Politiques d'action sociale auprès de la population immigrante étrangère en Espagne: approximations du schéma d'intervention à partir du travail social », Nouvelles pratiques sociales, vol. $14, \mathrm{n}^{\circ} 1$, p. 183-207.

Agrela, B. (2002). Spain as a Recent Country of Immigration: How Immigration Became a Symbolic, Political, and Cultural Problem in the "New Spain", San Diego, University of California.

Alscher, S. (2005). Knocking at the Doors of "Fortress Europe": Migration and Border Control in Southern Spain and Eastern Poland. Working paper 126. San Diego, Center for Comparative Immigration Studies, University of California.

Bailly, E., et L. Licata (2011). «De l'ombre à la lumière, du déni à la reconnaissance : Une approche psychosociale de l'implication des sans-papiers dans des mouvements d'occupations d'églises ». Alexandries, $n^{0} 24$, en ligne :

[http://www.reseau-terra.eu/article1193.html].

Bergeron, J., et S. Potter (2006). Family members and relatives an important resource for newcomer's settlement. Canadian Issues, printemps, p. 76-80.

Castello, C. (2013). «Las grandes empresas confían en que la economía española se estabilice ». Cinco Dias, 2 janvier, en ligne :

[http://cincodias.com/cincodias/2013/01/02/empresas/1357297136_850215.html].

Cohen-Émerique, M. (1993). «L'approche interculturelle dans le processus d'aide », Revue santé mentale au Québec, vol. 18, n 1, p. 1-19.

Cohen-Émerique, M. (2000). «L'approche interculturelle auprès des migrants ». Dans G. Legault (dir.), L'Intervention interculturelle, Boucherville, Gaëtan Morin, p. 161-184.

Cruz Roja Española (CRE) (2006). Acciones para la inclusión: Buenas prácticas y talleres de participación. Madrid, Cruz Roja Española.

Cruz Roja Española (CRE) (2011). Memoria explicativa 2011 proyecto: intervención socioeducativa y intercultural con menores extranjeros no acompañados y acompañamiento de jóvenes extutelados en riesgo social. Grenade, Cruz Roja Española.

Cruz Roja Española (CRE) (2012). Conócenos. En ligne :

[http://www.cruzroja.es/portal/page?_pageid=638,12410926\&_dad=portal30\&_schema=POR TAL30].

Fong, R. (2004). « Culturally Competent Contextual Social Work Practice and Intersectionality ». Dans R. Fong (dir). Culturally Competent Practice with Immigrant and Refugee Children and Family, New York, The Guilford Press, p. 309-313.

Gomez Gil, C. (2005). Las ONG en España, de la apariencia a la realidad. Madrid, Los libros de la Catarata. 
Hopkins, P., et M. Hill (2010). « The needs and strengths of unaccompanied asylum-seeking children and young people in Scotland ». Child and Family Social Work, vol. $15, n^{\circ} 4$, p. 399408.

HCNUR (2009). Separated children in Europe program. Statement of good practice. $4^{\mathrm{e}}$ édition, Bruxelles.

Jimenez, M. (2007). Una Mirada Transnacional: Contextos migratorios en Marruecos de los menores migrantes e intervencion en origen. Communication présentée au Colloque International de la migration des mineurs non accompagnés en Europe, octobre 2007, Poitiers, France.

Kohli, R. (2006). «The Sound of Silence: Listening to what unaccompanied asylum-seeking children say and do not say », British Journal of Social Work, vol. 36, n 5, p. 707-721.

Kohli, R., et Mitchell, F. (2007). Working with unaccompanied asylum seeking children: Issues for policy and practice, Basingstoke (UK) et New York (NY), Palgrave Macmillan

Kwok, S.M., et D. M.Y. Tam (2009). «Chinese Immigrant Youth and the Justice System in Canada », Canadian Social Work, vol. 12, n 1, p. 114-122.

Legault, G. (dir.) (2000). L'intervention interculturelle. Boucherville, Gaëtan Morin.

Lévesque, J., et J. Panet-Raymond (1994). "L'évolution et la pertinence de l'approche structurelle dans le contexte social actuel », Service social, vol. 43, nº 3, p. 23-29.

Ministerio de Empleo y Seguridad social, (2013). Autorización de residencia temporal de menores no acompañados. En ligne :

[http://extranjeros.empleo.gob.es/es/InformacionInteres/InformacionProcedimientos/Ciudadan osnocomunitarios/hoja062/index.html].

Moffette, D. (2009). Menaces à la frontière. Problématisations de l'(im)migration irrégulière à Ceuta, enclave espagnole au nord du Maroc. Mémoire de maîtrise en anthropologie, Université Laval, Québec. En ligne :

[http://webcache.googleusercontent.com/search?q=cache:OktuAYrbG1YJ:www.theses.ulaval .ca/2009/26390/26390.pdf+\&cd=1\&hl=fr\&ct=clnk\&gl=ca].

Montgomery, C. (2002). «The Brown Paper Syndrome: Unaccompanied minors and questions of Status », Refuge, vol. 20, n 4, p. 56-67.

Moreau, M. (1987). «L'approche structurelle en travail social : implications pratiques d'une approche intégrée conflictuelle », Service social, vol. 36, $\mathrm{n}^{\text {os }} 2-3$, p. 227-245.

Observatorio de la infancia en Andalucía (OIA) (2006). Buenas Practicas en la atención a menores inmigrantes en Andalucía. Grenade, Junta de Andalucía.

Observatorio de la infancia en Andalucía (OIA) (2009). Cifras y datos: Menores Extranjeros (No. 6). En ligne : [http://www.observatoriodelainfancia.es/oia/esp/cifras_y_datos.aspx].

Observatorio de la infancia en Andalucía (OIA) (2012). Inmigración: Estado de la infancia y adolescencia en Andalucía. Cuaderno 2. Grenade, Junta de Andalucía. 
Potocky-Tripodi, M. (2002). Best Practices for Social Work with Refugees and Immigrants, New York, Colombia University Press.

Senovilla, D. (2007). Situación y tratamiento de los menores extranjeros no acompañados en Europa. Un estudio comparado de 6 países: Alemania, Bélgica, España, Francia, Italia y Reino Unido. Bélgica: Observatorio Internacional de Justicia Juvenil.

Setien, M-L., et F. Barcelo (2007). El tratamiento de los menores extranjeros no acompañados en Europa: El caso español. Communication présentée au Colloque International de la migration des mineurs non accompagnés en Europe, octobre 2007, Poitiers, France.

Suárez Navaz, L. (2004). « Niños entre fronteras: migración de menores no acompañados en el mediterráneo occidental », Migración y Desarrollo, n² 2, p. 35-48.

Vatz-Laaroussi, M. (2009). Mobilité, réseaux et résilience. Le cas des familles immigrantes et réfugiées au Québec, Québec, Presse de I'Université du Québec.

Verbunt, G. (2009). La question interculturelle dans le travail social. Repères et perspectives, Paris, La Découverte.

Wade, J. (2011). «Preparation and transition planning for unaccompanied asylum-seeking and refugee young people: A review of evidence in England », Children and Youth Services Review, vol. 33, $n^{\circ} 12$, p. 2424-2430.

Wouk, J., Y. Soojin, L. Roach, J. Thomson et A. Harris (2006). « Unaccompanied/Separated minors an refugee protection in Canada: Filling Information Gaps », Refuge, vol. 23, $\mathrm{n}^{\circ} 2$, p. $125-136$. 